


\title{
Thermal Lens Spectrometry to Study Complex Fluids
}

\author{
M. L. Baesso ${ }^{1}$, J. R. D. Pereira ${ }^{1,2}$, A. C. Bento ${ }^{1}$, A. J. Palangana ${ }^{1}$, \\ A. M. Mansanares ${ }^{2}$, and L. R. Evangelista ${ }^{1}$ \\ ${ }^{1}$ Departamento de Física, Universidade Estadual de Maringá, \\ Avenida Colombo, 5790, Maringá, Paraná, Brazil \\ ${ }^{2}$ Instituto de Física Gleb Wataghin, Universidade Estadual de Campinas, \\ Unicamp, 13083-970, Campinas, SP, Brazil
}

Received 26 October, 1998

\begin{abstract}
We describe applications of Thermal Lens Spectrometry to study optical and thermal properties of lyotropic liquid crystal. In particular, we refer to methods for measurements of thermal diffusivity anisotropy at different temperatures and as a function of the ferrofluids doping. The theoretical basis for quantitative measurements is discussed together with the advantages and limitations as compared with conventional methods. Finally, future developments of photothermal techniques for these applications are assessed.
\end{abstract}

PACS numbers: 61.30.-v 61.30.Gd 65.70.+y

\section{Introduction}

The importance of studying the properties of complex fluids cannot be underestimated. For the liquid crystal families there are many important applications which are crucial in everyday life, such as the displays, electrooptical components, sensing, and so on. The liquid crystals have called the attention of many scientists of different areas because these systems present the absolutely unique flow phenomena which are very difficult to understand since they combine the flow properties of liquids with the orientational order of crystals driving the induced mass motion to an specific pattern which depends on both the applied forces into the system and their physical and chemical properties. For example, the application of external fields induces symmetry changes with consequent modification on their texture and basic properties, such as the values of the elastic constant, magnetic susceptibility, refractive index, viscosity, etc. [1-6] Laser induced refractive index changes have been widely employed in order to understand the liquid crystal systems [7-16]. By changing the beam power, experimental configuration and time scale of the experiments, many optically induced nonlinear mechanisms have been investigated [7]. In all of these previous studies, the experiments and theoretical mod- els have been applied in thermotropic liquid crystal in order to obtain their fundamental properties. Thermal Lens Spectrometry(TLS) is a photothermal technique which is based on the change in the refractive index of the sample when it is illuminated by a laser beam. Recently, we have introduced this technique in the study of lyotropic liquid crystals $[17,18]$. TLS method was employed in order to measure the thermal diffusivity anisotropy of the nematic phase of this family of liquid crystals. The geometrical form of the micelles was correlated to the anisotropy of the heat diffusion in the two directions of the samples. Subsequent works [19-22] have been devoted to the study of the role of ferrofluid in the lyotropic system, but a definitive description of the composed sample thermal anisotropy still needs to be presented.

Photothermal techniques are advantageous as compared to conventional thermometry because they usually allow the experiments to be performed without the dificulties of obtaining the steady state conditions. Near phase transitions, photothermal methods allow a better definition of the critical temperature, since in the calorimetric methods and thermal flow techniques the experiments have been made with thermal gradients higher than $10^{\circ} \mathrm{C}([8,23])$. Inherent in these scientific 
studies is the need to determine rates of heat diffusion into and through such a system which in turn requires measurements of physical-chemical properties of the obtained device. Although lyotropic liquid crystals have not yet been used for technological applications, their structure presents similarities with many biological systems [25] and therefore their basic properties may be useful for the understanding of these related systems. Although being desirable, it is not often possible to make bulk or interfacial measurements of the thermal properties of this system because of the need to probe non-destructively and non-invasively the sample. It is for this reason that photothermal techniques are promising for the study of this system since they are non-contacting, sensitive, remote and easy to perform [25,26-31].

The aim of this paper is to discuss some of the recent advances in the thermal lens methods with special reference to the measurements of thermo-optical properties of lyotropic systems. In particular, the modemismatched thermal lens method will be described in order to analyze the quantitative measurements in undoped and ferrofluid doped samples.

\section{Theoretical basis of thermal lens spectrometry}

All photothermal techniques are based in the use of electromagnetic radiation to induce non-radiative deexitation of excited states resulting in a change in temperature of the tested sample. This temperature rise can be measured through different processes. Many of the techniques use laser as the exitation beam. This brings advantages such as: high power density, coherence, directional and spatial stability, the possibility of probing very small volumes and the ability of performing non-contacting measurements [27].

TLS is one of the photothermal techniques which is remote and quite sensitive. The TL effect is created when the excitation beam passes through the sample and the absorbed energy is converted into heat. The consequent change in the refractive index induced by a temperature rise will produce a lenslike optical element at the sample, the so-called thermal lens. The propa- gation of a probe beam through the TL will be affected resulting in a spreading or focusing of the beam centre. By measuring its beam center intensity in the far field, the optothermal properties of the sample can be determined. Since the first report of the thermal lens effect [32] the sensitivity of the technique has been improved by changing the experimental configuration. The modemismatched TLS method has been demonstrated to be the most sensitive [26]. In this case the sample is positioned at the waist of the excitation beam, where the power density is maximum, Fig. 1. In these arrangement a $\mathrm{TEM}_{00}$ gaussian beam illuminates a sample, causing a thermal lens. A weak probe beam passes through the the sample to probe the thermal lens. The position of the waist of the probe beam is taken as the origin along the $Z$ axis. The sample is located at $Z_{1}$ and the detector plane is positioned at $Z_{1}+Z_{2}$. The waist of the probe beam is $\omega_{o p}$, the radius of the probe beam and the excitation beam in the sample are $\omega_{o p}$ and $\omega_{e}$, respectively. The theoretical treatment for the thermal lens effect needs to take into account realistic boundary conditions of the sample in both radial and axial directions. Their theoretical basis have been developed and presented in the references $[28,29,31,33,34]$. A brief review of this three-dimensional model will be presented in this work, with special emphasis in the approaches necessary for the liquid crystal measurements.

There are three steps in deriving the aberrant model [33]. First, the temperature rise in the sample induced by the optical energy absorption is calculated. Second, the phase shift of the probe beam caused by the refractive index change is obtained and finally, the Fresnel diffraction theory is used to calculate the field distribution of the probe beam in the detector plane. The model uses the following considerations: both the excitation and the probe beam lasers are Gaussian and in the $\mathrm{TEM}_{00}$ mode; The sample is homogeneous and obey the Beer's Law; detection of the probe beam is obtained in the Fresnel region.

\section{A. Temperature rise in the sample}

The differential equation of conduction of heat in an isotropic sample can be written as[29, 38]

$$
\frac{\partial}{\partial t}\left[\Delta T\left(r_{1}, \phi_{1}, z, t\right)\right]-D \nabla^{2}\left[\Delta T\left(r_{1}, \phi_{1}, z, t\right)\right]=\frac{Q\left(r_{1}, \phi_{1}, z, t\right)}{c \rho} .
$$




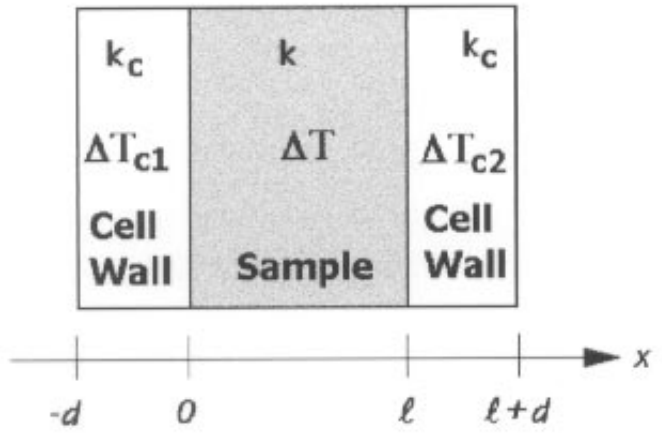

Figure 1: Scheme of the geometric position of the beams in a mode-mismatched dual beam TL experiment.

In Eq. (1) $\left(r_{1}, \phi_{1}, z\right)$ are the cylindrical coordinates in the sample; $\Delta T\left(r_{1}, \phi_{1}, z, t\right)$ is the temperature rise in the sample $(\mathrm{K}) ; Q\left(r_{1}, \phi_{1}, z, t\right)$, the heat source, is the heat generated per unit volume and unit time $\left(\mathrm{J} \mathrm{s}^{-1}\right.$ $\left.\mathrm{cm}^{-3}\right) ; D=k / c \rho$ is the thermal diffusivity of the sample $\left(\mathrm{cm}^{2} \mathrm{~s}^{-1}\right)$, where $k$ is the thermal conductivity (W $\left.\mathrm{cm}^{-1} \mathrm{~K}^{-1}\right), c$ the specific heat $\left(\mathrm{J} \mathrm{g}^{-1} \mathrm{~K}^{-1}\right)$, and $\rho$ the density $\left(\mathrm{g} \mathrm{cm}^{-3}\right)$ of the sample; $t$ is time $(\mathrm{s})$. The temperature rise can be obtained by solving this heat conduction equation with suitable heat source and appropriate boundary conditions.

In the TL experiment the heat source in Eq. (1) is the absorbed excitation energy by the sample assuming that all of the energy is converted to heat by nonradiative processes. The $\mathrm{TEM}_{00}$ Gaussian laser beam can be expressed as [35-39]

$$
I_{e}(r)=\frac{2 P_{e}}{\pi \omega_{e}^{2}} \exp \left(-\frac{2 r^{2}}{\omega_{e}^{2}}\right)
$$

where $I_{e}(r)$ and $P_{e}$ are the intensity $\left(\mathrm{W} \mathrm{cm}^{-2}\right)$ and total power (W) of the excitation beam, respectively. The heat generated due to the optical absorption per unit volume and unit time at $\left(r_{1}, z\right)$ in a medium with optical absorption coefficient $A\left(\mathrm{~cm}^{-1}\right)$, is then [36, 37]

$$
Q\left(r_{1}, z\right)=A I_{e}\left(r_{1}\right) \exp (-A z) .
$$

The 3D temperature distribution treatment takes realistic axial boundary condition, shown in Fig. 2, into account, while the radius of the sample is assumed as infinite. In Fig. 2, the thickness of the sample cell wall is $d(\mathrm{~cm})$, and its conductivity is $K_{c}\left(\mathrm{~W} \mathrm{~cm}^{-1} \mathrm{~K}^{-1}\right)$. $\Delta T_{c 1}$ and $\Delta T_{c 2}$ are the temperature rises in the cell walls. The boundary conditions are that temperature rises and heat fluxes are continuous at the boundaries, $z=-d, 0, l$, and $l+d$. Neglecting the heat capacity of the cell wall, we have

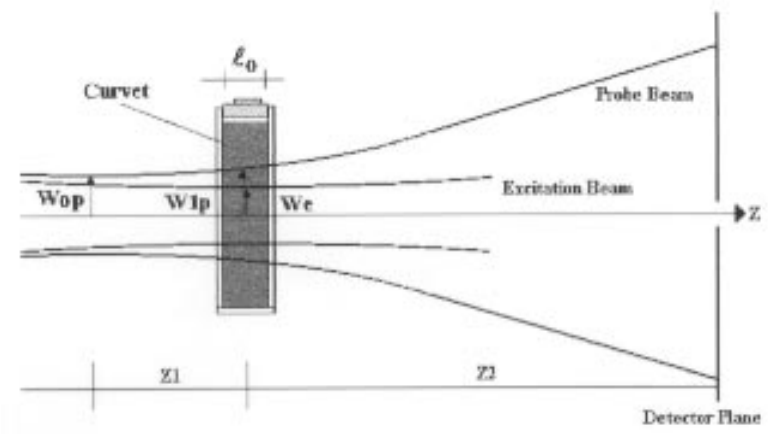

Figure 2: A schematic diagram of the sample and sample cell.

$$
\left|\frac{\partial}{\partial z}\left[\Delta T_{c i}\left(r_{1}, z, t\right)\right]\right| \simeq\left|\frac{\Delta T_{c i}\left(r_{1}, z, t\right)-0}{d}\right|, \quad(i=1,2 ; z=0, l,
$$

providing the temperature rise in air is zero. Considering the heat flux directions at the boundaries, the boundary condition can be expressed as $[29,36,38]$

$$
\frac{\partial}{\partial z}\left[\Delta T\left(r_{1}, z, t\right)\right]=h\left[T\left(r_{1}, z, t\right)\right], \quad\left(r_{1}<\infty, z=0, l\right),
$$

where $h=k_{0} /(k d)$.

The Green's function yielding the temperature rise at $\left(r_{1}, \phi_{1}, z\right)$ and time $t$ for a unit heat source at $\left(r_{1}^{\prime}, \phi_{1}^{\prime}, z^{\prime}\right)$ and time zero in the sample with the boundary condition, Eq. $(5)$, is given $($ see $[32,38])$ as

$$
G\left(r_{1}, r_{1}^{\prime}, \phi_{1}, \phi_{1}^{\prime}, z, z^{\prime}, t\right)=\frac{e^{-R^{2} /(4 D t)}}{2 \pi D t} \sum_{n=1}^{\infty} \frac{A_{n}(z) A_{n}\left(z^{\prime}\right)}{l\left(\alpha_{n}^{2}+h^{2}\right)+2 h} e^{-D \alpha_{n}^{2}}
$$

with 


$$
A_{n}(\zeta)=\alpha_{n} \cos \left(\alpha_{n} \zeta\right)+h \sin \left(\alpha_{n} \zeta\right)
$$

In Eqs. (6) and (7) $\alpha_{n}$ represents the positive roots of

$$
\tan (\alpha l)=\frac{2 \alpha h}{\alpha^{2}-h^{2}}
$$

whereas

$$
R^{2}=r_{1}^{2}+{r_{1}^{\prime}}^{2}-2 r_{1} r_{1}^{\prime} \cos \left(\phi_{1}-\phi_{1}^{\prime}\right) .
$$

The temperature rise in the sample is then

$$
\Delta T\left(r_{1}, z, t\right)=\int_{0}^{l} \int_{0}^{l} \int_{0}^{\infty} \int_{0}^{2 \pi} \frac{Q\left(r_{1}^{\prime}, z^{\prime}\right)}{c \rho} G\left(r_{1}, r_{1}^{\prime}, \phi_{1}, \phi_{1}^{\prime}, z, z^{\prime}, t\right) r_{1}^{\prime} d \phi_{1}^{\prime} d r_{1}^{\prime} d z^{\prime} d t^{\prime}
$$

Substitution of Eqs. (3) and (6) into Eq. (10) yields

$$
\begin{aligned}
\Delta T\left(r_{1}, z, t\right) & =\frac{P_{e} A}{\pi t_{c} k} \sum_{n=1}^{\infty} \frac{\frac{y_{n}}{l} \cos \left(\frac{y_{n} z}{l}\right)+h \sin \left(\frac{y_{n} z}{l}\right)}{y_{n}^{2} / l+l h^{2}+2 h} \\
& \times F_{n} \int_{0}^{t} \frac{1}{1+2 t^{\prime} / t_{c}} \exp \left[-\frac{2 r_{1}^{2} / \omega_{e}^{2}}{1+2 t^{\prime} / t_{c}}-D\left(\frac{y_{n}}{l}\right)^{2} t^{\prime}\right] d t^{\prime},
\end{aligned}
$$

where

$$
y_{n}=\alpha_{n} l,
$$

and

$$
F_{n}=\frac{\exp (-A l)}{A^{2}+\left(y_{n} / l\right)^{2}}\left[\left(\frac{y_{n}^{2}}{l^{2}}-A h\right) \sin \left(y_{n}\right)-\frac{y_{n}}{l}(A+h) \cos \left(y_{n}\right)\right]+\frac{y_{n}(A+h)}{\left.A^{2} l+y_{n}^{2} / l\right)} .
$$

Furthermore, in Eq. (11)

$$
t_{c}=\frac{\omega_{e}^{2}}{4 D}
$$

is a characteristic thermal time constant.

\section{B. Phase shift of the probe beam}

The refractive index $n\left(r_{1}, z, t\right)$ of the sample is a function of temperature as

$$
n\left(r_{1}, z, t\right)=n_{0}+\frac{d n}{d T} \Delta T\left(r_{1}, z, t\right),
$$

where $n_{0}$ is the refractive index at initial temperature. When the probe beam propagates through the TL its wave front is slightly distorted, and the distortion can be expressed as an additional phase shift, which can be found by considering the problem from the point of view of optical path length variation regarding the axis [35].

The phase shift $\Phi\left(r_{1}, t\right)$, therefore, is [35,36]

$$
\Phi\left(r_{1}, t\right)=\frac{2 \pi}{\lambda_{p}} \int_{0}^{l}\left[n\left(r_{1}, z, t\right)-n(0, z, t)\right] d z,
$$

where $\lambda_{p}$ is the wavelength of the probe beam. Substituting Eqs. (11) and (15) into Eq. (16) gives 


$$
\begin{aligned}
\Phi\left(r_{1}, t\right) & =\frac{2 \beta}{t_{c}} \sum_{n=1}^{\infty} \frac{\sin \left(y_{n}\right)-\left(h l / y_{n}\right) \cos \left(y_{n}\right)+h l / y_{n}}{y_{n}^{2} / l+l h^{2}+2 h} F_{n} \\
& \times \int_{0}^{t} \frac{1}{1+2 t^{\prime} / t_{c}}\left\{\exp \left[-D\left(\frac{y_{n}}{l}\right)^{2} t^{\prime}\right]-\exp \left[-\frac{2 r_{1}^{2} / \omega_{e}^{2}}{1+2 t^{\prime} / t_{c}}-D\left(\frac{y_{n}}{l}\right)^{2} t^{\prime}\right]\right\} d t^{\prime},
\end{aligned}
$$

where

$$
\beta=-\frac{P_{e} A}{\lambda_{p} k} \frac{d n}{d T} .
$$

The $\mathrm{TEM}_{00}$ Gaussian probe beam has emerged from the sample with the additional phase shift, (Eq.(17)) can then be expressed as $[33,35,39]$

$$
U_{p}\left(r_{1}, Z_{1}, t\right)=\left(\frac{2 P_{p}}{\pi}\right)^{1 / 2} \frac{1}{\omega_{1 p}} \exp \left[-j \frac{\pi}{\lambda_{1 p}}\left(2 Z_{1}+\frac{r_{1}^{2}}{R_{1 p}}\right)-j \Phi\left(r_{1}, t\right)-\frac{r_{1}^{2}}{w_{1 p}}\right],
$$

where $P_{p}$ and $R_{1 p}$ are the total probe beam power $(\mathrm{W})$ and the radius of the wave-front curvature of the probe beam at $Z_{1}$, respectively.

\section{Propagation of the probe beam}

The probe beam coming from the sample with the additional phase shift propagating to the detector plane can be treated as diffraction by using Fresnel diffraction theory [33,35,36], given as [40]

$$
\begin{aligned}
U_{p}\left(r_{2}, Z_{1}+Z_{2}, t\right) & =\frac{1}{\lambda_{p} Z_{2}} \exp \left[-j \frac{2 \pi}{\lambda_{p}}\left(Z_{2}+\frac{r_{2}^{2}}{2 Z_{2}}\right)\right] \\
& \times \int_{0}^{\infty} U_{p}\left(r_{1}, Z_{1}, t\right) \exp \left(-j \frac{\pi}{\lambda_{p}} \frac{r_{1}^{2}}{Z_{2}}\right) r_{1} d r_{1} G\left(r_{1}, r_{2}\right),
\end{aligned}
$$

with

$$
G\left(r_{1}, r_{2}\right)=\int_{0}^{2 \pi} \exp \left(j \frac{2 \pi}{\lambda_{P}} \frac{r_{1} r_{2}}{Z_{2}} \cos \left(\phi_{1}-\phi_{2}\right)\right) d \phi_{1}
$$

where $\left(r_{1}, \phi_{1}\right),\left(r_{2}, \phi_{2}\right)$ are the polar coordinates at the sample and at the detector plane, respectively. Substituting Eq. (19) into Eq. (20) and remembering that [41-43]

$$
\begin{gathered}
G\left(r_{1}, r_{2}\right)=2 \pi J_{0}\left(\frac{2 \pi}{\lambda_{p}} \frac{r_{1} r_{2}}{Z_{2}}\right), \\
\omega_{1 p}^{2}=\omega_{0 p}^{2}\left[1+\left(Z_{1} / Z_{c}\right)^{2}\right],
\end{gathered}
$$

and

$$
R_{1 p}=\left(Z_{1}^{2}+Z_{c}^{2}\right) / Z_{1}
$$

for the Gaussian probe beam, where

$$
Z_{c}=\pi \omega_{0 p}^{2} / \lambda_{p}
$$

is the confocal distance of the probe beam, we have the complex amplitude of the probe beam at the detector plane as 


$$
U_{p}\left(r_{2}, Z_{1}+Z_{2}, t\right)=C \int_{0}^{\infty} \exp [-(1+j \nu) g-j \Phi(g, t)] J_{0}\left(\frac{2 \pi \omega_{e} r_{2}}{\lambda_{p} Z_{2}} \sqrt{m g}\right) d g
$$

where

$$
\begin{gathered}
\nu=\frac{Z_{1}}{Z_{c}}+\frac{Z_{c}}{Z_{2}}\left[1+\left(\frac{Z_{1}}{Z_{c}}\right)^{2}\right], \\
C=j \frac{\omega_{1 p} \sqrt{2 \pi P_{p}}}{\lambda_{p} Z_{2}} \exp \left[-j \frac{2 \pi}{\lambda_{p}}\left(Z_{1}+Z_{2}+\frac{r_{2}^{2}}{2 Z_{2}}\right)\right], \\
g=\left(r_{1} / \omega_{1 p}\right)^{2},
\end{gathered}
$$

and

$$
m=\left(\omega_{1 p} / \omega_{e}\right)^{2},
$$

which is the degree of mismatching of the probe beam and excitation beams in the sample. Here $J_{0}$ is the zerothorder Bessel function. In Eq. (26) the phase shift of the probe beam is expressed as

$$
\begin{aligned}
\Phi(g, t) & =\frac{2 \beta}{t_{c}} \sum_{n=1}^{\infty} \frac{\sin \left(y_{n}\right)-\left(h l / y_{n}\right) \cos \left(y_{n}\right)+h l / y_{n}}{y_{n}^{2} / l+l h^{2}+2 h} F_{n} \\
& \times \int_{0}^{t} \frac{1}{1+2 t^{\prime} / t_{c}}\left\{\exp \left[-D\left(\frac{y_{n}}{l}\right)^{2} t^{\prime}\right]-\exp \left[-\frac{2 m g}{1+2 t^{\prime} / t_{c}}-D\left(\frac{y_{n}}{l}\right)^{2} t^{\prime}\right]\right\} d t^{\prime} .
\end{aligned}
$$

Finally, the probe beam intensity distribution $I_{p}$ at detector plane is given as

$$
I_{p}\left(r_{2}, Z_{1}+Z_{2}, t\right)=\left|U_{p}\left(r_{2}, Z_{1}+Z_{2}, t\right)\right|^{2},
$$

which can be simplified as $I_{p}(t)$ when only the center point of the probe beam at the detector plane is considered. When $m=1$, which is a mode-matched dual-beam or single-beam TLS situation, Eq. (26) becomes the same form as that obtained by Wu and Dovichi's[36].

In ref. [29] the comparison between $3 \mathrm{D}$ finite and 2D conventional infinite models is made, in which a 2D infinite conventional model for cw laser-induced mode-mismatched dual-beam TLS (Ref. [34]) is used, as:

$$
\begin{gathered}
\Delta T_{1}\left(r_{1}, t\right)=\frac{P_{e} A}{2 \pi k t_{c}} \int_{0}^{t} \frac{1}{1+2 t^{\prime} / t_{c}} \exp \left(-\frac{2 r_{1}^{2} / \omega_{e}^{2}}{1+2 t^{\prime} / t_{c}}\right) d t^{\prime}, \\
\Phi_{I}\left(r_{1}, t\right)=\frac{\theta}{t_{c}} \int_{0}^{t} \frac{1}{1+2 t^{\prime} / t_{c}}\left[1-\exp \left(-\frac{2 r_{1}^{2} / \omega_{e}^{2}}{1+2 t^{\prime} / t_{c}}\right)\right] d t^{\prime}, \\
U_{p I}\left(r_{2}, Z_{1}+Z_{2}, t\right)=C \int_{0}^{\infty} \exp \left[-(1+j \nu) g-j \Phi_{I}(g, t)\right] J_{0}\left(\frac{2 \pi \omega_{e} r_{2}}{\lambda_{p} Z_{2}} \sqrt{m g}\right) d g
\end{gathered}
$$

and

$$
I_{p I}(t)=I_{p I}(0)\left[1-\frac{\theta}{2} \tan ^{-1}\left(\frac{2 m \nu}{\left[(1+2 m)^{2}+\nu^{2}\right]\left(t_{c} / 2 t\right)+1+2 m+\nu^{2}}\right)\right]^{2},
$$

where

$$
\theta=\beta l
$$

and $I$ denotes the $2 \mathrm{D}$ infinite model. 


\section{Experimental}

Thermal lens measurements can be performed for both time-resolved and steady state mode. Up to now, in the study of liquid crystal, we have used time-resolved methods and the experimental arrangement shown in Fig. 3. The excitation laser beam is a $6 \mathrm{~W} \mathrm{Ar}^{+}$laser (Coherent Innova 90 Plus ) at $514.5 \mathrm{~nm}$ or at $488 \mathrm{~nm}$. The sample was put at the focal plane of the lens 1 and its expossure to the excitation beam was controlled by a shutter. The probe beam was an He-Ne laser at $632 \mathrm{~nm}$. The output of the photodiod was coupled to a storage oscilloscope. The magnitude of the thermal lens signal with time was recorded and the data thus obtained were processed using least-squares curve fit.

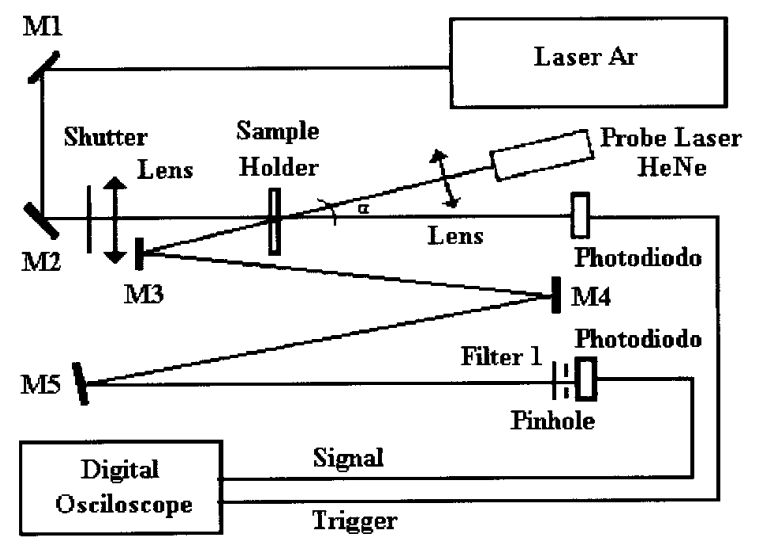

Figure 3: Mode-mismatched thermal lens experimental setup.

The Lyotropic nematic liquid crystals studied were: sample 1) potassium laurate(29.4 wt\%), decanol (6.6 wt\%), water (64 wt\%); sample 2) potassium laurate $(34.5$ wt\%), potassium chlorate $(3 \mathrm{wt} \%)$, water $(62.5$ wt\%); sample 3) sample 1 doped with 0.08 wt\% of ferrofluid and sample 4 doped with 0.34 wt\% of ferrofluid. Ferrofluid enhance alignment of the micelles in the presence of a magnetic field. The phase sequences of the samples were determined by optical microscopy and conoscopic observation. The axes of the directors were aligned in a magnetic field for both orientations, paralell and perpendicular to the side walls. The measurements were performed at room temperature and also for different temperatures [17-19,21,22].
IV Thermal diffusivity measurements

Fig. 4 shows the experimental data and the theoretical best curve fitting of Eq. (36) for sample 3 with the micelles aligned parallel to the side walls. As it can be seen the signal intensity decreases with time, which means that $d n / d T<0$. Table I shows the thermal difusivity results for the samples investigated in both, perpendicular and parallel directions of the director. For the undoped samples the ratios between the thermal diffusivity parallel and perpendicular $D_{\|} / D_{\perp}$ are around 1.2. In all of the results, the ratios between $D_{\|} / D_{\perp}$ are smaller than those measured for thermotropic liquid crystal, obtained by several techniques, which are about 2.0. In order to explain this difference we have considered the sample as an array of thermal resistor of rode-like shape and developed the following equation $[44,45]$

$$
\frac{D_{\|}}{D_{\perp}}=\frac{L(1+2 S) / R+(2-2 S)}{L(1-S) / R+(2+S)}
$$

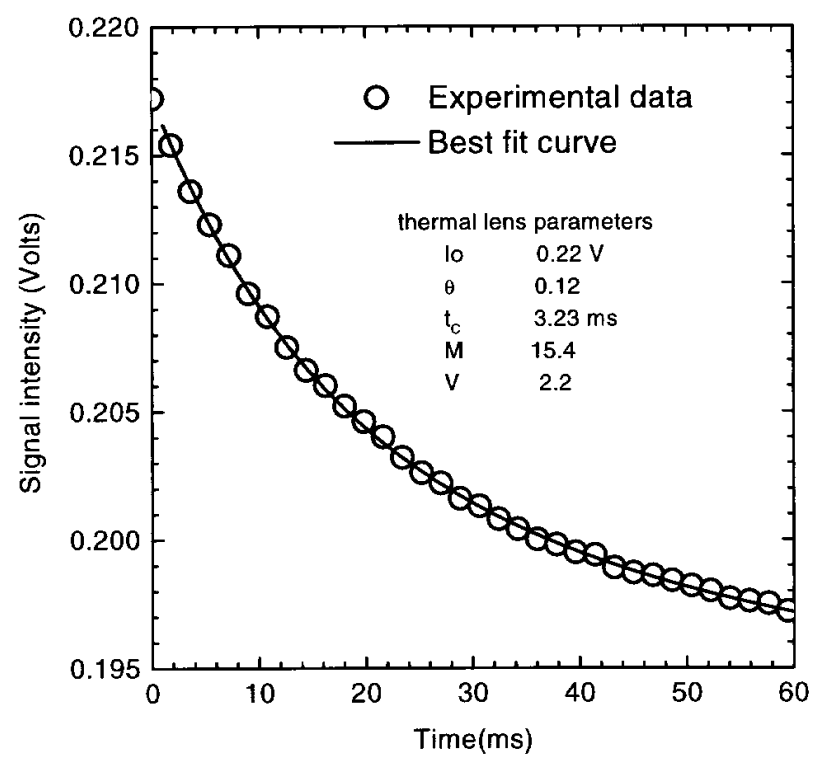

Figure 4: Thermal lens experimental data of sample 3 with the director aligned parallel to the sidewalls and their best fit curve. 
Tabela 1: Thermal diffusivity paralell and perpendicular for the ferrofluid doped lyotropic liquid crystal

\begin{tabular}{c|c|c}
\hline $\begin{array}{c}\text { ferrofluid content } \\
\text { (wt.\%) }\end{array}$ & $\begin{array}{c}\alpha_{\|} \\
\left(10^{-3} \mathrm{~cm}^{2} / \mathrm{s}\right)\end{array}$ & $\begin{array}{c}\alpha_{\perp} \\
\left(10^{-3} \mathrm{~cm}^{2} / \mathrm{s}\right)\end{array}$ \\
\hline 0 & $1.69 \pm 0.05$ & $1.40 \pm 0.03$ \\
\hline $0.080 \pm 0.008$ & $1.40 \pm 0.09$ & $1.35 \pm 0.13$ \\
\hline $0.290 \pm 0.029$ & $1.58 \pm 0.04$ & $1.54 \pm 0.05$ \\
\hline $0.340 \pm 0.034$ & $1.25 \pm 0.09$ & $1.44 \pm 0.14$ \\
\hline $0.4 \pm 0.04$ & $1.67 \pm 0.1$ & $1.75 \pm 0.04$ \\
\hline
\end{tabular}

In Eq. (38) $S$ is the scalar order parameter and $L$ and $R$ are the length and diameter of the cylinders respectively. Lyotropic liquid crystals in the nematic calamitic phase are mixtures composed of micelles with geometrical anisotropy shapes of about 1.6, while thermotropic liquid crystals molecules length-to-width ratio is about $3.0[46,47]$. Using the above expressions we could find the estimated value of $D_{\|} / D_{\perp}=1.3$ for lyotropic samples and $D_{\|} / D_{\perp}=1.9$ for thermotropic ones. In this treatment the thermal diffusivity anisotropy was associated to the form of the micelles for lyotropic nematics and the molecular shape for thermotropic liquid crystals, as predicted by Eq. (38). In contrast, for ferrofluid doped samples, this treatment does not explain the thermal diffusivity anisotropy values. For both samples it can be observed, Table I, that the anisotropy decreases as ferrofluid concentration increases (see also Fig. 5).

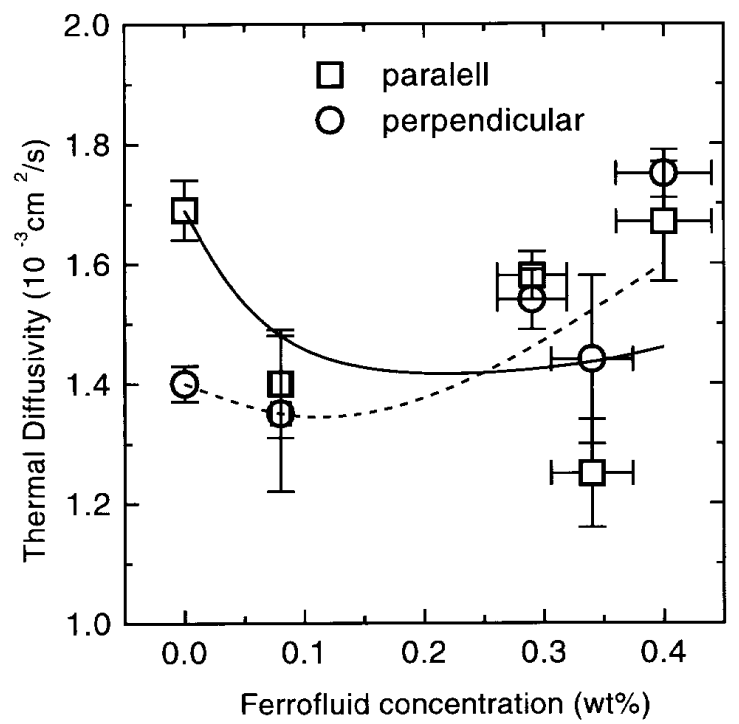

Figure 5: Parallel and perpendicular thermal diffusivities as a function of ferrofluid concentration.
Furthermore, the values of the thermal diffusivity decreases as ferrofluid concentration increases. These results suggest that ferrofluid particles may play as an addition thermal resistence in the sample and their conection to the order parameter still need to be clarified.

\section{Future work}

The ultimate goal in the study of thermal diffusivity of lyotropic liquid crystal using photothermal technique is the determination of the role played by the ferrofluid particles in the heat diffusion process in both directions of the sample. Based on recent results in this system $[19,21,22]$ we have suggested that ferrofluid particles may induce an additional thermal barrier in the sample. These experiments were performed for both changing ferrofluid concentration and the sample temperature. However, further studies to obtain a more detailed physical description of the thermal anisotropy of this doped liquid crystal are still required. This seems to be a difficult task, since the experimental discrimination between the laser beam intensity change induced by both the doping and the liquid crystal itself is difficult. Furthermore, there are concentration gradient across the beam which may not be linear. In this way it is necessary to determine the gradient profile of the doping. This may be possible to investigate through time resolved measurements since the thermal diffusivity and gradient diffusion coefficients are usually at least two order of magnitude different.

For experiment in function of temperature, thermal lens seems to be a valuable technique to measure the thermo-optical parameters of liquid crystal[22]. Fig. 6 shows the sample heater that can be used for such measurements. This is possible to be make because of the especial ability of the technique of being a remote method. In addition the change in the temprerature induced by excitation beam at the sample is lower than $10^{-2}{ }^{\circ} \mathrm{C}$, allowing therefore a fine determination of the experimental parameters around the sample phase transitions. An additional application of thermal lens spectrometry in the study of liquid crystals is the determination of the absolute values of $d n / d T$. This is an important parameter because it contains information 
about sample thermal expansion, polarizability, refractive index, viscosity, etc. The study of $d n / d T$ in liquid crystal is also attracting due to the its unusual behavior. It has been observed in thermotropic liquid crystal, that the refractive index change can produce either laser beam self-focussing and defocusing, depending on the sample orientation and the laser beam polarization.

The use of TLS as quantitative method to study liquid crystal through the tree-dimensional model reviewed in this work needs to obey some experimental conditions, such as performing the measurements for a short period of time and with the ratio between the probe and excitation beam spot sizes at the sample no larger than 3 . This procedure avoid heat diffusion in the laser beam direction, which is not taken into account in the development of the model. For thin sample, such as liquid crystal, this heat flow can invalidate the obtained results.

In summary, with the introdution of thermal lens spectrometry, to study lyotropic liquid crystal we have investigated the lyotropic systems difusion rates and tried to associate the anisotropy of the thermal diffusivity with their structure. The method used is promissing, since it is very sensitive, remote, non-cantacting and allows the determination of the absolute values of the sample thermo-optical parameters.

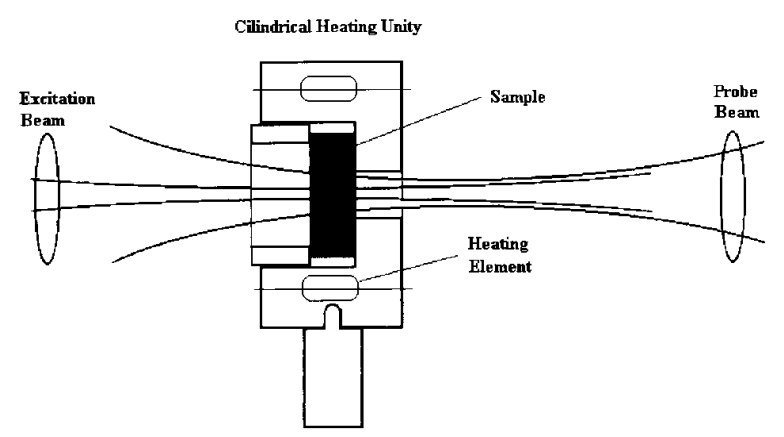

Figure 6: Sample holder and heating unity for the modemismatched thermal lens measurement.

\section{Acknowledgments}

We are gratefull to the Brazilian Agencies CNPqPADCT (Contract n. 620810/95-5), FAPESP and CAPES for the financial support of this work.

\section{References}

[1] P.G. de Gennes, The Physics of Liquid Crystals, (Clarendon, Oxford, 2nd edition, 1993).

[2] S. Chandrasekhar, Liquid Crystals, (Cambridge University, Cambridge, 1977)

[3] A.J. Palangana, L.R. Evangelista and A.K. Zvezdin, Phys. Lett. A 200, 56(1995).

[4] M. Simões, A.J. Palangana, and L.R. Evangelista. Phys. Rev. E 54, 3765(1996).

[5] A.J. Palangana, M. Simões, L.R. Evangelista and A.A. Arrotéia, Phys. Rev. E 56, 4282 (1997).

[6] M. Simões, A.J. Palangana and L.R. Evangelista, Phys. Rev. E 56, 7326 (1997).

[7] I.C. Khoo and S.T. Wu, Optics and Nonlinear Optics of Liquid Crystals (World Scientific, Singapore, 1983).

[8] G. Koren, Phys. Rev. A 13, 1177 (1976).

[9] I.C. Khoo, S.L. Zhuang and S. Shepard, Appl. Phys. Lett. 39, 937 (1981)

[10] R. MacDonald and H.J. Eichler, Appl. Phys. B 60, 543 (1995).

[11] Y.G. Fuh and R.F. Code, Can. J. Phys. 63, 282 (1985)

[12] I.C. Khoo, Phys. Rev. A 25, 1040 (1982).

[13] V. Volterra and E. Wiener-Avnear, Opt. Commun. 12, 194 (1974)

[14] N.F. Pilipetski, A.V. Sukhov, N.V. Tabiryan, and B. Ya. Zel'dovich, Opt. Commun. 37, 280 (1981).

[15] I. Jánossy, Phys. Rev. E 49, 2957 (1994).

[16] R. Muenster, M. Jarasch, X. Zhuang and Y.R. Shen, Phys. Rev. Lett. 78, 42 (1996).

[17] A.C. Bento, A.J. Palangana, L.R. Evangelista, M.L. Baesso, J.R.D. Pereira, E.C. da Silva, A.M. Mansanares, Appl. Phys. Lett. 68, 3371(1996).

[18] J.R.D. Pereira, Dissertação de Mestrado, IFGWUnicamp, 1997.

[19] J.R.D. Pereira, A.M. Mansanares, A.J. Palangana, M.L. Baesso. Submitted to Mol. Cryst. Liq. Cryst. (1998).

[20] S.M. Shibili and L.L. Dantas, Appl. Phys. Lett. 72, 674 (1998).

[21] J.R.D. Pereira, A.M. Mansanares, A.J. Palangana, M.L. Baesso in preparation.

[22] J.R.D. Pereira, A.M. Mansanares, A.J. Palangana, M.L. Baesso in preparation.

[23] F. Mercuri, U. Zammit and M. Marinelli, Phys. Rev. E 57, 596 (1998).

[24] P.J. Coolings, Liquid Crystals: Nature's Delicate Phase of Matter, (Princeton University Press, Princeton, 1991).

[25] T. Higaishi, T. Imasaka and N. Ishibashi, Ann. Chem. 55, 1907 (1983).

[26] F.J. Power and E.D. Salin, Ann. Chem. 60, 838 (1988).

[27] R.D. Snook, R.D. Lowe and M.L. Baesso, Analyst, 23 587 (1998). 
[28] M.L. Baesso, A.C. Bento, A.A. Andrade, J.A. Sampaio, E. Pecoraro, L.A.O. Nunes, T. Catunda, and S. Gama, Phys. Rev. B 57, 10545 (1998).

[29] J. Shen, M.L. Baesso and R.D. Snook, J. Appl. Phys. 75, 3738 (1994).

[30] M.L. Baesso, J. Shen and R.D. Snook, Chem. Phys. Lett. 197, 255 (1992).

[31] M.L. Baesso, J. Shen and R.D. Snook. J. Appl. Phys. 75, 3732 (1994).

[32] J.P. Gordon, R.C.C. Leite, R.C. Morre, S.P.S. Porto and J.R. Whinnery, J. Appl. Phys. 36, 3 (1965).

[33] J. Shen, R.D. Lowe and R.D. Snook, Chem. Phys. 165, 385 (1992).

[34] J. Shen and R.D. Snook, J. Appl. Phys. 73, 5286 (1993).

[35] S.J. Sheldon, L.V. Knight and J.M. Thorne, Appl. Opt. 21, 1663 (1982).

[36] S. Wu and N.J. Dovichi, J. Appl. Phys. 67, 1170 (1990).

[37] F.W. Dabby, R.W. Boyko, C. Shank and J.R. Whinnery, IEEE J. Quantum Electron. QE-5, 516 (1969).
[38] H.S. Carslaw and J.C. Jaeger, Conduction of Heat in Solids, (Clarendon, Oxford, 2nd edition, 1959).

[39] A.E. Sigmen, An Introduction to Lasers and Masers (McGraw-Hill, New York, 2nd edition, 1971).

[40] A.K. Ghatak and K. Thyagarajan, Contemporay Optics, (Plenum, New York, 1978).

[41] N.W. McLachlan, Bessel Functions for Engineers, (Clarendon, Oxford, 2nd edition, 1961).

[42] R.C. Weast, Handbook of Chemistry and Physics (CRC, Boca Roton, FL, 67th edition, 1986-1987).

[43] A.J. Chapman, Heat Transfer (Macmillan, New York, 2nd edition, 1960).

[44] G. Vertogen and W.H. de Jeu, Thermotropic Liquid Crystals, (Verlag, Berlin, 1988).

[45] K.S. Chu and D.S. Moroi, J. Phys. Colloq. 36, C1-99 (1975).

[46] Y. Hendrix, J. Charvolin, M. Rawiso, L. Liébert and M. C. Holmes, J. Phys. Chem. 87, 3991 (1983).

[47] A.V.A. Pinto and L.Q. Amaral, J. Phys. 94, 3186 (1990). 